\title{
L'invalidation du facteur de transcription GATA-2 chez la souris perturbe l'hématopoièse
}

La différenciation des cellules hématopoiétiques se poursuit tout au long de la vie. Au départ se trouvent les cellules souches, peu nombreuses, capables de se renouveler et de produire les progéniteurs de l'ensemble des cellules. Dans l'embryon de mammifère, le siège de l'hématopoïèse se déplace du sac vitellin (hématopoïèse primitive) au foie fotal puis à la moelle osseuse (hématopoïèse définitive). Une telle évolution est contrôlée par les effets combinés de facteurs de croissance et de facteurs de transcription nucléaire, capables d'activer des gènes spécifiques pour chaque lignage, et qui déclenchent ou arrêtent le cycle cellulaire. Trois membres d'une famille de facteurs de transcription, dits GATA-1, 2 et 3 se présentent comme candidats à la régulation de l'expression génique dans les cellules hématopoiétiques.

Les facteurs GATA ont été ainsi nommés parce qu'ils se lient à une séquence consensus centrée sur les nucléotides GATA, présente dans de nombreuses régions régulatrices de gènes dont les premiers découverts ont été ceux de $\beta$ et $\alpha$ globine ; ils contiennent un ou deux motifs à doigt de zinc. Le premier de ces facteurs transcriptionnels s'est appelé, successivement, GF-1, NF-E1, Erifl, puis GATA-1 [ 1, 2]. Son importance a pu être prouvée par des expériences d'invalidation du gène chez la souris en 1991 ( $/ \mathrm{s} n^{\circ} 4$, vol. 7, p. 385). Les meurent avant la date de naissance normale dans un tableau où domine l'anémie. Trois autres protéines, GATA-2, 3 et 4, ont rejoint le premier membre du groupe. GATA-4 est spécifique du muscle cardiaque $(\mathrm{m} / \mathrm{s}$ $n^{\circ} 11$, vol. 10, p. 1161), GATA-3 est surtout abondante dans les cellules lymphoïdes T, tandis que GATA-2 est exprimée dans les progéniteurs précoces et dans les cellules érythroïdes jeunes, les mastocytes, les mégacaryocytes. Une équipe de Boston, MA, USA [3] a identifié l'ADNc de GATA-2 ; il mesure $2,6 \mathrm{~kb}$ et code pour une protéine de 470 acides aminés. Pour tenter de préciser le rôle et l'importance de GATA-2 dans l'hématopoïèse, l'équipe de Boston dirigée par S. Orkin a entrepris des essais d'invalidation, symétriques de ceux réalisés sur GATA-1 [4]. L'inactivation a suivi une voie désormais classique. Une cassette neo remplaça un domaine à doigt de zinc nécessaire pour la liaison avec l'ADN, rendant la protéine néoformée inactive. Des clones de cellules $\mathrm{ES}$, hétérozygotes $\mathrm{GATA}^{+} /-$puis homozygotes $\mathrm{GATA}^{-} /^{-}$, furent réimplantés à des souris normales, et furent à l'origine de souris hétérozygotes. Celles-ci se révélèrent normales. En revanche, lorsqu'on croisa entre elles ces souris hétérozygotes, aucune souris homozygote pour le déficit ne fut obtenue sur 73 naissances. En examinant de plus près les embryons, on constata qu'aucun homozygote ne dépasse 11,5 jours embryonnaires (jE).
Ces embryons apparaissent grossièrement normaux, mais le sac vitellin est pâle et presque vide. A 9,5 jE, les embryons sont encore vivants, de taille comparable à celle des témoins ; toutefois ils sont pâles, et le nombre des cellules sanguines est fortement réduit.

Pour examiner de plus près la contribution des souris GATA-2- /- $^{-}$aux diverses lignées sanguines, les auteurs ont préparé des chimères en insérant des cellules ES déficientes dans des blastocystes normaux. A $13 \mathrm{jE}$ (hématopoïèse primitive) on constate la présence de cellules négatives dans le sang, bien qu'en nombre diminué. A $17 \mathrm{jE}$ il n'y a plus de contribution des cellules GATA-2- $/^{-}$dans le sang, bien que leur niveau soit élevé dans le cerveau. Après la naissance, les tissus hématopoiétiques ne contenaient pas de cellules GATA- $2^{-} /{ }^{-}$. Ces cellules subissent un déficit sélectif concernant l'hématopoïèse définitive. Il concerne toutes les lignées produites par la moelle osseuse, et atteint également les cellules lymphoïdes. Les résultats de Tsai et al. [4] mettent en valeur le rôle central de GATA-2 dans l'hématopoï̀se: en son absence, l'hématopoïèse primitive du sac vitellin n'est pas supprimée mais affaiblie au point d'empêcher la survie au-delà de $11 \mathrm{jE}$. L'hématopoïèse définitive est presque complètement abolie. Le facteur agit à un stade très précoce, cellules souches ou progéniteurs jeunes, et gouverne la production de tous les li- 
gnages. Comme les propriétés de GATA-1, sans être identiques, sont très comparables, la question se pose des relations entre les deux facteurs. Les cellules GATA- $2^{-} /^{-}$expriment une quantité normale de messager GATA-1, l'action de GATA-2 ne passe donc pas par GATA-l. Réciproquement, GATA-1 réprime l'expression de GATA-2 lors de la maturation érythroïde [2].

Le phénotype des embryons GATA$2^{-}{ }^{-}$peut être comparé à celui d'autres mutants de souris affectant l'hématopoïèse, qui sont en général létaux plus tardivement dans la vie embryonnaire, aux $15^{\mathrm{e}}-16^{\mathrm{e}} \mathrm{jE}$, tels que $\mathrm{c}^{-} \mathrm{myb} \mathrm{C}^{-}$- ou $\mathrm{Rb}^{-} /^{-}$. La situation la plus comparable semble être celle du système W-Steel, qui montre des mutations dans le récepteur ou le ligand kit (m/s $n^{\circ} 10$, vol. 6, p. 1016). Cependant l'effet de ces mutations est plus tardif et n'affecte pas tous les lignages.

Le blocage de l'hématopoìèse par l'absence de GATA-2 n'est pas absolument total car il peut être partiellement levé in vitro par l'addition d'un ensemble de facteurs de croissance. Enfin, il ne semble pas y avoir d'effet sur les autres tissus, même ceux dans lesquels GATA-2 est fortement exprimé.

Pour l'avenir, l'étude plus fine des régulations des réactions où intervient GATA-2 aux divers stades de la différenciation devrait à bref délai fournir des renseignements importants.

1. Yamamoto M, Ko LJ, Leonard MW, Beug H, Orkin S, Engel JD. Activity and tissue-specific expression of the transcription factor NF-El multigene family. Genes Dev $1990 ; 4$ : 1650-62.

2. Weiss M J, Keller G, Orkin S. Novel insights into erythroid development revealed through in vitro differentiation of GATA-1 embryonic stem cells. Genes Dev 1994 ; 8 : 1184-97.

3. Dorfman DM, Wilson DB, Bruns GAP, Orkins. Human transcription factor GATA-2. J Biol Chem $1992 ; 267: 1279-85$

4. Tsai FY, Keller G, Kuo FC, Weiss MJ, Chen J, Rosenblatt M, Alt FW, Orkin S. An early haematopoietic def ect in mice lacking the transcription factor GATA-2. Nature 1994 ; 371 : 221-6.

$\mathrm{m} / \mathrm{s} n^{\circ} 11$, vol. 10 , novembre 94

\section{BRÈVES}

Les récepteurs des immunoglobulines dans l'inflammation. La réaction d'Arthus est un cas d'hypersensibilité provoquée par des complexes immuns. Expérimentalement, une réaction d'Arthus passive indirecte est déclenchée par in jection de l'anticorps sous la peau et de l'antigène par voie intraveineuse. Les complexes immuns activent ainsi la voie classique du complément qui attire les polynucléaires vers les sites d'injection où ils relarguent les médiateurs de l'inflammation. La contribution des récepteurs des régions constantes d'immunoglobuline $(\mathrm{FCR})$ dans la réponse inflammatoire a pu être évaluée grâce à une lignée de souris ayant une délétion génétique de la sous-unité $\gamma$ des FcR. Ces souris n'expriment plus les FcR à forte et faible affinité des IgG (Fc $\gamma$ RI et $\mathrm{Fc} \gamma \mathrm{RIII})$ ni le récepteur à forte affinité des $\operatorname{IgE}(\mathrm{Fc \varepsilon RI})$. La réaction d'Arthus est induite avec de l'ovalbumine d'œuf de poule et des IgG spécifiques. Les estimations histologiques des œedèmes, des hémorragies et des infiltrations de neutrophiles montrent clairement que la réaction inflammatoire est quasiment absente chez les animaux déficients. L'absence de réaction inflammatoire est à rapprocher de l'absence de récepteur d'IgG car les souris uniquement déficientes pour les récepteurs d'lgE, FcReRI, se comportent comme les animaux normaux. Le rôle des récepteurs d'IgG n'étant plus à démontrer, il reste à identifier les cellules impliquées dans la réaction d'Arthus. Parmi les cellules intervenant dans l'inflammation et exprimant les récepteurs d'IgG, les mastocytes font figure de favoris. Inhiber l'interaction des complexes immuns avec les FcR devient donc une nouvelle stratégie pour contrôler les réactions inflammatoires, notamment dans les maladies auto-immunes.

[Sylvestre DL, et al. Science $1994 ; 26$ : 1095-8.]

Invalidation $\mathrm{du}$ gène $c$-mpl chez la souris. Maintenant que les gènes du récepteur de la thrombopoïétine, $c-m p l$, et de la thrombo- poiétine, TPO, ont été clonés [1,2], le système de régulation de la production de plaquettes peut être étudié en détail. Deux équipes de Genentech (San Francisco, CA, USA) rapportent les résultats de l'invalidation du gène c-mpl par insertion dans le troisième exon d'une cassette neo dans des cellules embryonnaires de souris, puis injection de ces cellules dans des blastocystes murins [3]. La transmission par la lignée germinale a permis d'obtenir par croisement des souris homozygotes c-mptF. Celles-ci sont viables et d'apparence normale, leur seule anomalie apparente est une diminution de $85 \%$ du compte des plaquettes qui ont, de plus, un volume augmenté de $30 \%$. La moelle et la rate sont fortement déplétées de mégacaryocytes. Les animaux hétérozygotes n'ont pas d'anomalie plaquettaire. Les autres lignées hématopoiétiques sont normales, qualitativement et quantitativement. Enfin, l'activité TPO est très élevée chez les souris c-mptץ- alors qu'aucun autre facteur de croissance hématopoiétique n'est altéré. La raison pour laquelle $15 \%$ des plaquettes et des mégacaryocytes sont encore présents n'est pas claire : un récepteur tronqué s'insère-t-il en partie dans la membrane, ou la différenciation mégacaryocytaire peutelle être partiellement activée par d'autres cytokines? Ces résultats confirment cependant que c-mpl règle directement la prolifération et la maturation des mégacaryocytes, comme l'annonçaient la présence et l'expression de $c$-mpldans les cellules souches médullaires primitives [4]. L'altération phénotypique chez les souris c- $m p t^{-}$- est spécifique puisque seuls les mégacaryocytes et les plaquettes sont touchés; l'effet thérapeutique de TPO ne devrait donc s'exercer que sur ces cellules.

[1. Wendling F, Tambourin P. médecine/sciences 1991; 7 : 569-77.]

[2. Wendling $\mathrm{F}$, et al. médecine/sciences $1994 ; 10: 874-6$.]

[3. Gurney AL, et al. Science 1994; 265: 1445-7.]

[4. Méthia N, et al. Blood 1993; 82 : 1395-401.] 